\title{
Special Food Safety Issues during Pregnancy ${ }^{1}$
}

Claudia Peñuela and Amy Simonne 2

Pregnant women require a diet with essential nutrients for the health of the mother and her baby. During pregnancy women and the fetus are especially more susceptible to three types of foodborne agents: Listeria monocytogenes (causing listeriosis), methylmercury, and Toxoplasma gondii (causing toxoplasmosis).

\section{Listeriosis}

Listeriosis is a disease caused by Listeria monocytogenes, a bacterium commonly found in the environment which can then contaminate foods. Listeria monocytogenes can be transmitted to an unborn baby even if there are no illness symptoms for the mothers. In the first trimester, listeriosis may cause miscarriage. If contracted later in the pregnancy the disease may result in premature labor, low-weight infants or stillbirth. Listeriosis is most commonly contracted during the third trimester when the mother's immune system is weakened.

Listeria can grow well at refrigerated temperatures while most other bacteria do not. Specific foods that you must AVOID to prevent listeriosis:

- Unpasteurized (raw) milk and dairy products or any food made with unpasteurized milk.

- Soft cheeses such as feta, Brie, Camembert, blue-veined cheeses, or some "Mexican" cheeses such as "queso fresco" or "queso blanco," made with unpasteurized milk.

It is okay to eat soft cheeses if they are made with pasteurized milk. Read the label carefully!

- Ready-to-eat meat products such as hot dogs, luncheon meat, bologna, or other types of deli meats.

It is okay to eat these types of meats if they are reheated until steaming hot.

- Refrigerated pâtés and meat spreads.

- Smoked seafood, often found at the deli counter, or in the refrigerator section at the grocery store. Examples include smoked salmon, trout, whitefish, cod, tuna, or mackerel (all commonly labeled as "nova-style," "lox," "kippered," "smoked" or "jerky"). These still need to be heated or cooked to bring them to safe temperature levels.

You can eat canned or shelf-stable pâtés, meat spreads, salmon, tuna, and smoked seafood. Remember to refrigerate them after opening.

- Refrigerated ready-to-eat foods such as salads that are not cooked before eating.

1. This document is FCS8886, one of a series of the Department of Family, Y outh and Community Sciences, Florida Cooperative Extension S ervice, Institute of Food and Agricultural Sciences, University of Florida. First published: December 2009. Visit the EDIS Web site at http://edis.ifas.ufl.edu.

2. Claudia Peñuela, nutrition assistant-EFNEP, and Amy Simonne, Ph.D., associate professor, both of the Department of Family, Youth and Community Sciences; Institute of Food and Agricultural Sciences; University of Florida; Gainesville 32611. 


\section{Methylmercury}

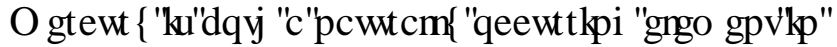
UXHHQYLRQP HQWOCAIDE SLRGXFWRICOXUWDOD

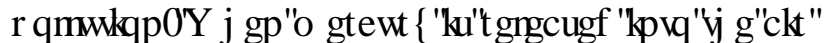

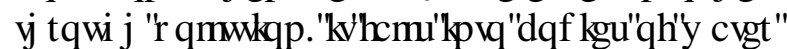

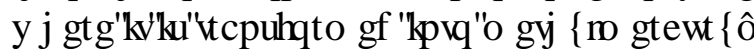

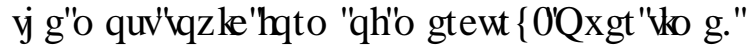
P HM Ф HFXU IIILFFX X(D)GIQMXIP DUQH

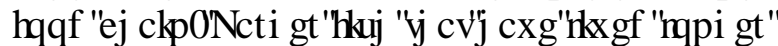

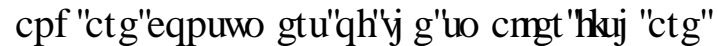

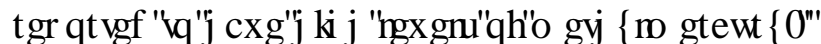
(]

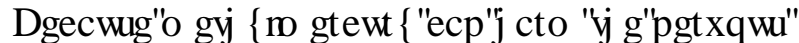

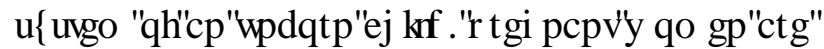

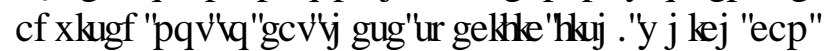

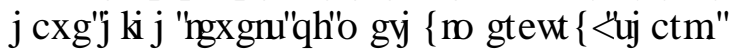
VZ RLALMUINDIP DFNHHDIDOAVILKU [) \&KHFNORFDODGYLUUHIIRUMDHFRQNX SWRQRI ILKKFDX KWQ RXUCRFDDHDVIS U RRGIHARXIFH LUMHC QYIRQP HQMOB LRMFWRQ\$ J HQF INUMMDW

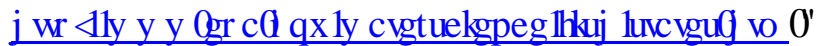
(]

( DUDJ IFRRNHGILMIMDRRGJ LYHM RXIKHDOW EHQHLWGXUQ $\square S U H$ QDQF $\mathbb{E} \backslash$ LSLRYGQ $\square R P$ H D

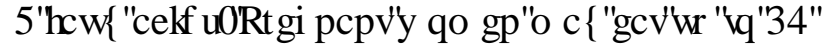
RXQFHVSHUZ HNRICQRZ IP HFXU ILMUDOA VHRRGIV RZIP HFXU IFKRIFHUAFOXGHWUP SW

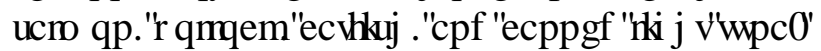

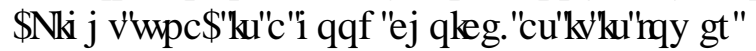

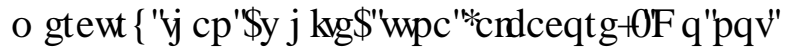
HDVP RUHMDOQ IRXQFHMSHUZ HNRIIEDFRH पZ KIMIXODIए

]

\section{Toxoplasmosis}

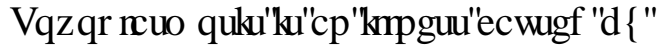

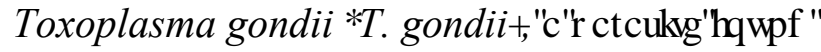
IQIDQXP EHURIMUQJ VIQFOXOQ IIXQFRRNHGIRU

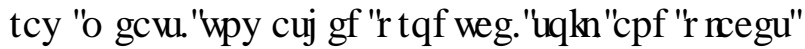

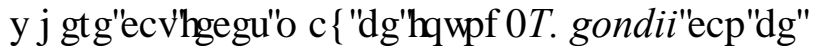
GHDOQ RIDQXXERQQEDE IRQFHUXHFHQWD QHURXVWK WMP [KDVEHQGHYHRSHGT\%DEIHV

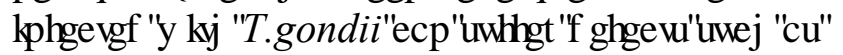

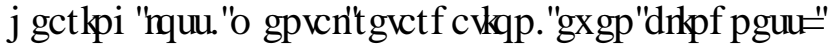

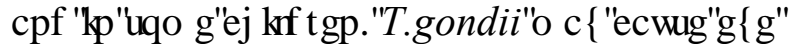

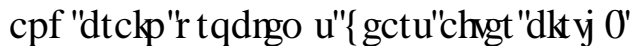
(] 7 RISLHHQW RSQDP RVW
[ ' RTQRWHDWWZ RRUXOCHFRRNGGP HDW HSHFIDO LSRULDP EUIDOGYHQLRQD

( ' RIQRWHDWXQZ DKHGIIUIWOQG YH HMEOHD

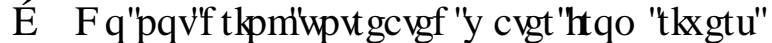
RUSRXQGVRUIQOMVGHYORSHGFRXQMUH] WLWP D $\square$ FRQMQQT.gondii $\square$

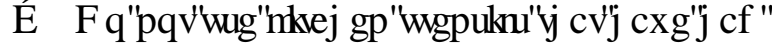
FRQMFWZ IWUD $\mathbb{P}$ HDW

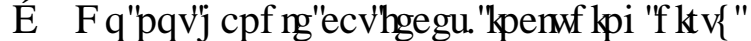

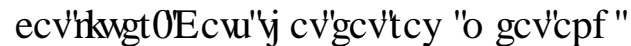
VP DODQIP DOVITIRI WQIKRWWRI $\square$. gondii WOA MLVSDWMMIFDQEHIIRXQGRQ

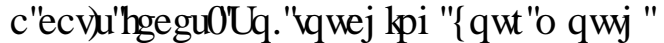

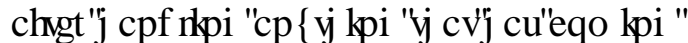
IQMRIFRQMFW LMKFDWHFHIFDQFDXM

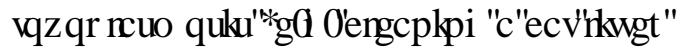
ER[ प⿴囗十

]

References

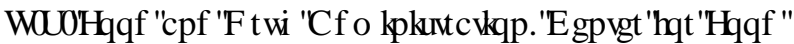
6DHWWOCAS SSQHG1 XWWRQMQGWIFood safety for moms-to-be $\square 2$ QWOHES YD(DECHIDW

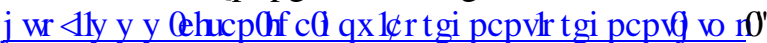
(

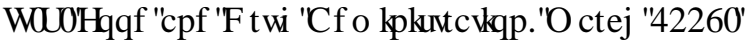

What You Need to Know About Mercury in Fish and Shellfish. $\gg 2$ QODOH

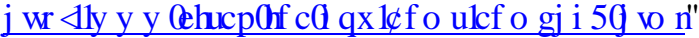
[

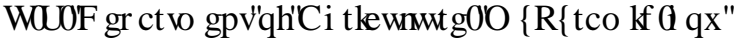
पQGपाMyPyramid for Pregnancy \& Breastfeeding Food Safety: Keep you and your baby safe from

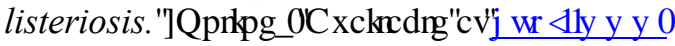
$\underline{P} \backslash S \backslash$ UP IGJ RYIP $\backslash S \backslash$ UP IAP RP VIRRGBWHWB OQWMURUVIKWI ()

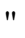

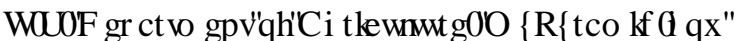
पQGपाMyPyramid for Pregnancy \& Breastfeeding Food Safety: Keep you and your baby safe from toxoplasmosis. ए2 Q0DH

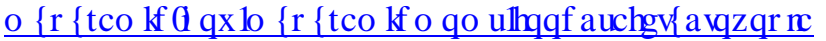
VP RUVIKWI O [

8 [6ए HSDWA HQWRIS J UFXOXIHIO \3 \UP IGJ RY पQGपाMyPyramid for Pregnancy \& Breastfeeding Food Safety: Eating fish while you are pregnant or breastfeeding $\square 2$ QDOH $\underline{\mathrm{P} \backslash S \backslash \mathrm{UP} \text { IGJ RYIP } \backslash S \backslash \mathrm{UP} \text { IGP RP VIRRGBWDHMBILK }}$ KWO 